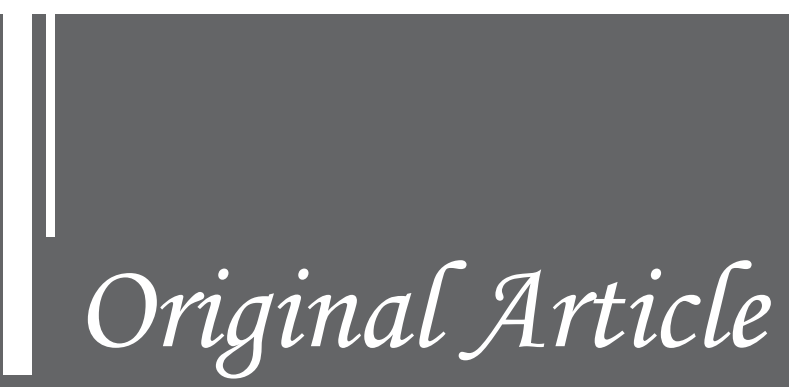

\title{
VITAMIN D DEFICIENCY AMONG DOCTORS AND STAFF NURSES: A NEGLECTED DOMAIN AMONG MEDICAL CARE GIVERS
}

\author{
Aisha Khan Jadoon ${ }^{1}$, Fasiha Sohail', Sarosh Khan Jadoon ${ }^{3}$, Arzu \\ Jadoon $^{4}$
}

\section{'Ziauddin University Hospital}

${ }^{2}$ Assisstant professor, Department of Medicine, Ziauddin University Hospital

${ }^{3}$ Resident Medical Officer, Department of Medicine, Ziauddin University Hospital

${ }^{4}$ Resident Medical Officer, Department of Medicine, Ziauddin University Hospital

Correspondence: Aisha Khan Jadoon, aishajadoon@hotmail.com

\begin{abstract}
Background: Various factors lead to hypovitaminosis (decreased vitamin D levels in serum/blood), in general population, sunlight being one of the major factors. Doctors and staff nurses are particularly at risk due to long working hours indoors, which deprives them of adequate Ultraviolet light exposure

Methods: It was a prospective descriptive cross sectional study conducted at Ziauddin University Hospital, Keamari campus, from August 2018 to July 2019. The objective of this study was to determine the status of vitamin D deficiency among doctors and staff nurses working at Ziauddin University Hospital. All those doctors and staff nurses were included in the study, who were already advised by physicians to get serum vitamin D levels done for their symptoms.

Results: A total sample of 118 was collected. 56 doctors and 62 staff nurses participated in the survey. 85 out of 118 (72\%) subjects were either deficient or insufficient of vitamin $\mathrm{D}$.

Conclusion: Even though doctors and nurses are considered to have better understanding of vitamin D deficiency symptoms and morbidity it causes; still this study found out that majority of the healthcare providers working at Ziauddin hospital were deficient of this vitamin.

Keywords: Vitamin d deficiency; doctors; nurses; tertiary care hospital; Pakistan
\end{abstract}




\section{Introduction}

Vitamin D And Its Significance

Vitamin D belongs to a group of secosteroids that are fat-soluble and exist in the five forms starting from vitamin D1 to D5. For human beings D2 (ergocalciferol) and D3 (cholecalciferol) hold the most significance. Calcitriol (1,25 Dihydroxycholecalciferol) is found to be an active form of vitamin D in vivo. Vitamin D absorbs calcium and phosphate from the intestines.(1)

Various factors that may lead to hypovitaminosis include obesity, multiparity(multiple pregnancies), clothing factor, diet, air pollution, inadequate exposure to sunlight, malabsorption; and, in infants, less amount of vitamin D found in breast milk.(2),(3) In adults, vitamin D deficiency can cause osteomalacia, osteoporosis and reduced bone mineral density; thus elevating the chances of bone fractures.(1) It can invite a plethora of problems comprising of metabolic disorders, autoimmune conditions, psychiatric, respiratory(breathing), cardiovascular(heart) diseases and even cancers.(1),(4) In paediatric(children less than 14 years) age group, it can lead to rickets which is characterized as bowing of the legs.(3)

Vitamin D is a synthetic product which is predominantly absorbed by fortified foods. Vitamin D3 is synthesized in a few stages. First of all in the skin where 7-dehydrocholesterol reacts with ultraviolet light from the sun and later in the liver it is hydroxylated to 25-hydroxyvitamin D (25(OH)-vitamin D) and in the kidneys it is further converted to the metabolically active form, 1?, 25-dihydroxyvitamin $\mathrm{D}(1$ ?, $25-(\mathrm{OH})$ 2D). Most of the vitamin D which is measured in serum is of D3 type. The amount of vitamin D recommended for all infants, children, and adolescents to be consumed in a day is 400 to 600 IU.(4),(5),(6)

Hypovitaminosis D(Vitamin D deficiency)

It is an extremely common health problem all around the planet; it seems to be widely recognized as a pandemic.(7) Approximately one million people in the world have low levels of vitamin D. A survey conducted in 2007 revealed the prevalence in Canada to be about 70\%97\%.(4) It was rare in South Asia previously.(8) But unfortunately currently it has reached epidemic proportions in South Asia. Studies from different Asian countries; Tehran, China, India, Turkey, Lebanon, Jordan, Saudi Arabia, Iran and Tunisia gave an evidence of high prevalence of vitamin D deficiency (44-95\%), although all of them have adequate sunshine.(9) Researches from our neighbor country, India report $80-85 \%$ prevalence of vitamin D deficiency in their local population.(10)

Prevalence of vitamin D deficiency in Pakistan is found to be to be $74 \%$.(11) Karachi is located at $24^{\circ} \mathrm{N}$ latitude and $67^{\circ} \mathrm{E}$ Longitude Although the weather in Karachi is warm or hot throughout the year and there is abundant sunlight almost daily but the prevalence of Hypovitaminosis D was alarmingly elevated according to studies conducted in Karachi from 2009 to 2015, (60\% to 91.5\%) (9),(11),(12) and $88 \%$ in Islamabad as per the data from 2015.(7)

Any activity that leads to a limited sunlight exposure raises the risk of decreased vitamin D levels. Since, vitamin D synthesis is highly reliant on sunlight, people who live under the circumstances that compel them to spend less time outdoors, have adverse impacts on vitamin D status. Health care professionals are assumed to be knowledgeable about the subject hence are expected that they might be least affected by vitamin $\mathrm{D}$ deficiency. It is a major health problem worldwide with significant morbidity (disease). This study aims to find out the prevalence of Vitamin D deficiency among doctors and nurses working in a tertiary care hospital.

\section{Methodology}

The study was a prospective descriptive cross sectional study conducted at Ziauddin University Hospital, Keamari campus, from August 2018 to July 2019. Ziauddin Hospital Keamari campus is a 100 bedded tertiary care facility. It has around 60 doctors besides consultants and 85 staff nurses in total.

A sample size of 118 patients (56 doctors and 62 staff nurses) were included in the study that fulfilled the inclusion criteria. Post graduate doctors, medical officers and staff nurses were included in the study where as consultants, morbidly high BMI, the ones taking vitamin D supplements and with history of adrenal or gonadal disease, history of gastrointestinal resection or hysterectomy, any metabolic bone disease, pregnancy and lactation, parathyroid, malignancy, thyroid, hepatic and renal disease, malabsorption syndrome or chronic diarrhea were excluded.

A questionnaire was devised that included age, designation, daily working hours and test results of vitamin D levels from Ziauddin Hospital's laboratory.

25-OH Vitamin D levels were diagnosed as:

\section{Deficient: $\quad<20 \mathrm{ng} / \mathrm{ml}$ \\ Insufficient: $\quad 20-29 \mathrm{ng} / \mathrm{ml}$

Sufficient: $\quad 30-100 \mathrm{ng} / \mathrm{ml}$ \\ Potential toxicity: $\quad>100 \mathrm{ng} / \mathrm{ml}$}

Staff nurses and doctors were not asked to get themselves tested particularly for the survey. Those who were genuinely affected by symptoms and were advised by different consultants to get the test done were included in the study. That was the reason for a long time for sample collection.

Results

Total sample of 118 was collected. 56 doctors and 62 staff nurses participated in the survey. Rest either did not fulfill the criteria or did not get a blood test done.

Total 63(53\%) females and 55(47\%) males were included in the survey. Age of doctors and nurses was between 24 to 38 years. Daily working hours for staff nurses are 6 hours to 12 hours. All doctors do a 9 hour job daily and post graduates do an additional 32 hours on call duty every third or fourth day.

85 out of $118(72 \%)$ subjects were either deficient or insufficient of vitamin $\mathrm{D}$. The difference between vitamin $\mathrm{D}$ level values can be assessed by looking at the pie chart (figure I). 48 out of $62(56 \%)$ staff nurses and 39 out of $56(46 \%)$ doctors were vitamin D deficient/ insufficient. $\quad 55(65 \%)$ females and 30(35\%) males were vitamin D insufficient or deficient. Severity of vitamin D deficiency is illustrated in Table I.

Table 1: Severity of vitamin D deficiency in staff and doctors

\begin{tabular}{|l|c|c|}
\hline \multicolumn{1}{|c|}{ SEVERITY OF DEFICIENCY } & \% & VALUE \\
\hline Deficient: $<\mathbf{2 0} \mathbf{~ n g / m l}$ & 47 & 55 \\
\hline Insufficient: $\mathbf{2 0} \mathbf{- 2 9} \mathbf{~ n g / m l}$ & 25 & 30 \\
\hline Sufficient: $\mathbf{3 0} \mathbf{- 1 0 0} \mathbf{~ n g / m l ~}$ & 28 & 33 \\
\hline Total & 100 & 118 \\
\hline
\end{tabular}

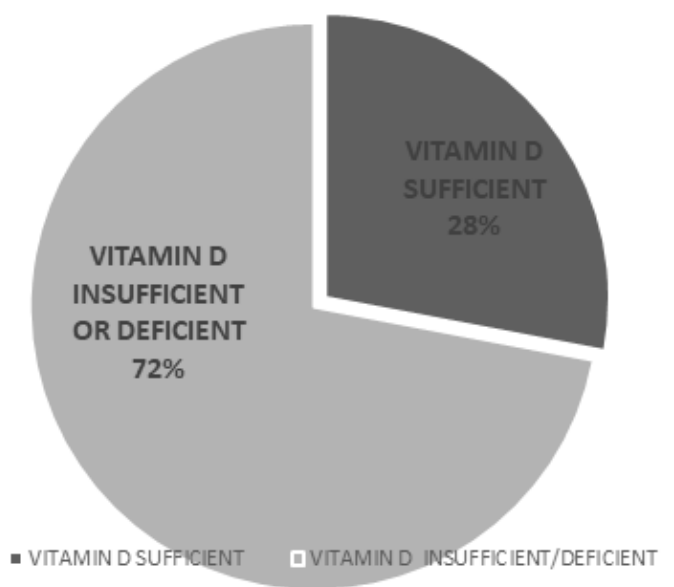

Figure 1: Proportion of vitamin D deficiency/insufficiency and sufficiency

\section{Discussion}

Vitamin D is most commonly estimated by the levels of 25hydroxyvitamin $\mathrm{D}$ that are circulating in the body. Serum level should be $>30 \mathrm{ng} / \mathrm{ml}(75 \mathrm{mmol} / \mathrm{l})$. It is further classified as insufficiency from 20 to $<30 \mathrm{ng} / \mathrm{ml}$, deficiency between $10 \mathrm{ng} / \mathrm{ml}$ and $20 \mathrm{ng} / \mathrm{ml}$, and severe deficiency $<10 \mathrm{ng} / \mathrm{ml}$. (13)

Literature from different countries, about vitamin D deficiency, clearly demonstrates magnitude of the issue but there are only few studies regarding this among health care professionals. To the best of authors' knowledge nothing as such has been carried out in Pakistan for the evaluation of vitamin D status among doctors or/and staff nurses.

Ziauddin Hospital as mentioned earlier is a 100 bedded tertiary care hospital with a busy ICU and 24 hours emergency department that 
provides state of the art facility to the less privileged population of Keamari.

Prevalence of reduced levels of vitamin D was enormously high, $72 \%$, among doctors and staff nurses in the current study. Almost half of the number of participants was vitamin D deficient and a quarter was insufficient, where as only $28 \%$ had normal levels of serum vitamin D.

A similar study was conducted on health professionals including doctors and staff nurses in Qatar, which revealed that $96.5 \%$ had vitamin $\mathrm{D}<30 \mathrm{ng} / \mathrm{ml},(14)$ Vitamin $\mathrm{D}$ deficiency has also been found in Indian health care workers.(10) According to a study carried out in 18 Indian cities, among medical and paramedical personnel, $79 \%$ of subjects were deficient, $15 \%$ were insufficient, and just $6 \%$ were sufficient in vitamin D status.(15) Medical staff reports of vitamin D from Iran showed $92 \%$ were deficient.(16) In Boston, 32\% healthy pupils, general physicians, and trainees were found to be vitamin D deficient.(17) In Portland, a study revealed that $51.4 \%$ internal medicine house staff was vitamin D deficient.(18)

Staff nurses in Ziauddin University Hospital work for 6 to 12 hours in a day. Most of them are rotated in the morning, evening and night shifts every 20 days. Doctors have to spend 9 hours daily in the hospital along with 32 hours on call every 3 rd or 4 th day as reported in this study. The possible reason for lower serum vitamin D levels among Medical care personnel can be a reduced exposure to sunlight. In general, workers who spent maximum time in an enclosed interior may be at jeopardy of vitamin D deficiency but there has been relatively little research into the healthiness of indoor workers.(19)

In this study females were found to be more vitamin D deficient as compared to males. One reason could be veiling or extra clothes for covering as per Islamic teachings and Pakistan's culture or avoiding tan but deficiency exists in both sexes. This suggests that veiling may influence degree of severity.(5) Shakiba M et al, supported the idea since females were more deficient in her study too.(16)

This study also found out that staff nurses were 10 times more at risk for vitamin D deficiency/ insufficiency as compared to doctors. Additional risk for staff could be demanding workload, shift working and the protective uniform; which limit the chance to have outdoor activity and sun exposure moreover demanding working condition also impairs the chances to have proper timely meals.

If the health care providers would have an idea about their own vitamin $\mathrm{D}$ levels, it might prove to be instrumental in changing their attitude towards vitamin D treatment in their patients. This study showed a surprisingly high prevalence of vitamin D deficiency and insufficiency among doctors and staff nurses. In order to improve the morbidity caused by it, serum vitamin D levels should be recommended to all doctors and nurses on regular basis.

There were certain limitations of this study. Firstly; it was not population-based so the results cannot be generalized. Secondly; it was a small sample size from a single hospital and lifestyle was not excluded.

\section{Conclusion}

Vitamin D deficiency/insufficiency is a worldwide awful predicament. The results of this study are an eye opener for all doctors and staff nurses and they should get their levels checked frequently and treat themselves. Health care professionals should take care of their own health to be adequately productive at work.

References

1. Daniel D, Bikle. Vitamin D Metabolism, Mechanism of Action, and Clinical Applications. Chem Biol. 2014 Mar 20;21(3):319-329.

2. T Durá-Travé, F Gallinas-Victoriano, M J ChuecaGuindulain, S Berrade-Zubiri. Prevalence of hypovitaminosis D and associated factors in obese Spanish children. Nutr Diabetes. 2017 Mar; 7(3): e248.
Umaretiya PJ, Oberhelman SS, Cozine EW, Maxson JA, Quigg SM, Thacher TD. Maternal Preferences for Vitamin D Supplementation in Breastfed Infants. Ann Fam Med. 2017 Jan; 15(1): 68-70.

4. Holick MF. Vitamin D deficiency. N Engl J Med. 2007;357(3):266-81.

5. Lehmann B, Meurer M. Extrarenal sites of calcitriol synthesis: the particular role of the skin. Recent Results Cancer Res. 2003;164:135-45.

6. Fuleihan G, Bouillon R, Clarke B, Chakhtoura M, Cooper C, McClung M, et al. Serum 25-hydroxyvitamin D levels: variability, knowledge gaps, and the concept of a desirable range. J Bone Miner Res. 2015; 30(7):1119-33

7. Kiani R A et al. Prevalence of Vitamin-D Deficiency in Urban Population: A Retrospective Analysis. Ann. Pak. Inst. Med. Sci. 2015; 11(2): 90-94

8. Kiani IG, Shah F, Mansur SS. Frequency of severe vitaminD deficiency in patients presenting to a tertiary care hospital in Islamabad. J Pak Med Assoc 2014; 46: 16-8.

9. Mahmood K, Akhtar ST, Talib A, Haider I. Vitamin-D status in a Population of Healthy Adults in Pakistan. Pak J Med Sci 2009; 25(4): 545-50.

10. Arya V, Bhambri R, Godbole MM, Mithal A. Vitamin D status and its relationship with bone mineral density in healthy Asian Indians. Osteoporos Int 2004; 15(1): 56-61.

11. Iqbal R, Jafri L, Haroon A, Khan AH. Illuminating the dark sidevitamin D status in different localities of Karachi. J Coll Physicians Surg Pak 2013; 23: 604-6.

12. Mehboobali N, Iqbal SP, Iqbal MP. High prevalence of vitamin D deficiency and insufficiency in a low income peri-urban community in Karachi. J Pak Med Assoc. 2015 Sep;65(9):946-49.

13. Chirita-Emandi A, Socolov D, Haivas C, Calapi? A Gheorghiu C, Puiu M (2015) Vitamin D Status: A Different Story in the Very Young versus the Very Old Romanian Patients. PLoS ONE 10(5): e 0128010 . https://doi.org/10.1371/journal.pone.0128010

14. Mahdy SM, Vitamin D status in health care professionals in Qatar. Saudi Med J 2010; Vol. 31 (1): 74-77

15. Beloyartseva $\mathrm{M}$ et al.Widespread vitamin $\mathrm{D}$ deficiency among Indian health care professionals, 2012, Volume 7, Issue 1-2, pp 187-192

16. Shakiba M, Rafiee P and Mohammadi A. High Prevalence of Vitamin D Deficiency in Medical Staff Draws Attention to a Retraining Program for Vitamin D. Austin J Nutri Food Sci. 2015;3(2): 1063.

17. Growdon Amanda S, Carlos A Camargo, Sunday Clark, Megan Hannon, Jonathan M Mansbach. Serum 25hydroxyvitamin d levels among Boston trainee doctors in winter. Nutrients. 2012; 4: 197-207.

18. Jancin B. Vitamin D deficiency common among physicians in training: long- term risk to bone health. Skin \& Allergy News2003; 34: 55 .

19. Mendell MJ, Fisk WJ, Kreiss K, Levin H, Alexander D, Cain WS, et al. Improving the health of workers in indoor environments: Priority research needs for a National Occupational Research Agenda. Am J Public Health. 2002;92(9):1430-40. 